\title{
Cohort-specific risk of suicide for different mental disorders in China
}

Yongsheng Tong and Michael R. Phillips

\section{Background}

The effect of gender, age, and urban versus rural residence on suicide risk among people with different mental disorders is unknown.

\section{Aims}

To estimate cohort-specific risk of suicide among people with different types of mental disorders using data from China's National Psychological Autopsy Study.

\section{Method}

Logistic regression models estimated adjusted odd ratios (OR) in 895 suicides and 701 injury deaths.

\section{Results}

The adjusted risk of suicide for mood disorders was highest $(\mathrm{OR}=44.2)$ followed by that for other mental disorders
$(\mathrm{OR}=10.7)$, anxiety disorders (OR $=10.2)$, psychotic disorders $(\mathrm{OR}=7.4)$, substance use disorders (2.7) and organic mental disorders $(O R=1.2)$. Risk of suicide among urban residents with any mental disorder was more than double that of rural residents $(\mathrm{OR}=15.5$ v. 7.6, $P=0.008)$.

\section{Conclusions}

The suicide risk associated with mental illness varies by type of illness and by demographic cohort. Lower risk of suicide among rural residents with mental disorders highlights the importance of non-mental health approaches to the prevention and management of suicidal behaviour in rural areas of low- and middle-income countries.

\section{Declaration of interest}

None.
Suicide is a leading cause of death throughout the world that is closely associated with mental illnesses. ${ }^{1-6}$ Given the importance of mental illness to suicide, some authors argue that reduction in mental health services leads to higher suicide rates, ${ }^{1}$ but other reports find that improved mental health services do not lead to decreased rates of suicide. ${ }^{7}$ One possible explanation for this discrepancy is that the risk of suicide varies by diagnostic group, so services may need to be more targeted to effectively reduce suicide rates. ${ }^{6,8}$ Another possibility is that the risk of suicide among people with specific types of mental disorders may vary by demographic group. For example, the risk of suicide among men with depression may be different from the risk among women with depression; if this were true, diagnostic-specific interventions would also need to be adapted to specific demographic cohorts to be most effective. Finally, urban versus rural residence - a factor that is not considered in current multidimensional models of suicide ${ }^{9-11}$ - may be an important moderator of the relationship between mental illness, mental health services and suicide, particularly in low- and middle-income countries with large agriculture-based rural populations.

We hypothesise that the risk of suicide varies by diagnostic group and that among individuals with specific types of mental disorders the risk of suicide varies by gender, age, and urban versus rural residence. China has a relatively high suicide rate and a unique profile of suicides - with much higher rates in rural than urban communities and slightly higher rates in women than in men ${ }^{4,5}$ - so this would be a good setting in which to investigate these relationships. The 1996-2000 National Psychological Autopsy Study ${ }^{5,12}$ collected a large sample of suicide decedents and non-suicidal injury death controls from 23 geographically representative regions of China, which is a suitable data-set to use to address this issue.

\section{Method}

The sampling methods have been described in detail in prior reports of the National Psychological Autopsy Study. ${ }^{5,12}$
Recruitment is shown in Fig. 1. Briefly, all deaths attributed to suicide and other injuries were investigated at 3 urban and 20 rural disease surveillance points, selected to be geographically representative of the 145 disease surveillance points in the national mortality surveillance system. The selected sample included 1854 people; 258 were excluded for various reasons (shown in Fig. 1). Among the remaining 1596 individuals, 853 were initially classified on the death certificate as suicides, 709 as specific types of injury deaths and 34 as accidental deaths of unknown cause. The cause of death was reclassified based on the detailed investigation of each decedent. This resulted in 895 suicide decedents (the case group) and 701 non-suicidal injury death decedents (the control group). The method of death among suicide decedents were as follows: $58 \%$ pesticide ingestion; $22 \%$ hanging; $4 \%$ each by medication ingestion, drowning and jumping; and $8 \%$ by other methods.

Most psychological autopsy studies employ living controls ${ }^{3,6}$ but we chose to use deceased controls because the methodological advantages of using deceased controls ${ }^{13}$ are particularly important for China. Except in rare cases where there is a possibility that the death may be as a result of murder, coroners' investigations are not conducted following suicides in China, so the only record of death is the death certificate. Only about $5 \%$ of individuals with mental illnesses in the country ever see a mental health professional, ${ }^{14}$ so mental health records are not available for most suicide decedents. In almost all cases the only available information is that provided by proxy informants. Thus when trying to identify risk factors for suicide it is essential to ensure the comparability of the proxy informants for cases of suicide with those for controls. Proxy informants for living controls report about an individual who they currently have regular contact with; this is very different from proxy informants for suicide decedents who report about a relative or associate who died unexpectedly several months previously. We chose accidental death decedents as our controls because proxy informants for these decedents have also experienced the recent death of the person they are reporting about. Selection of deceased controls could potentially result in 


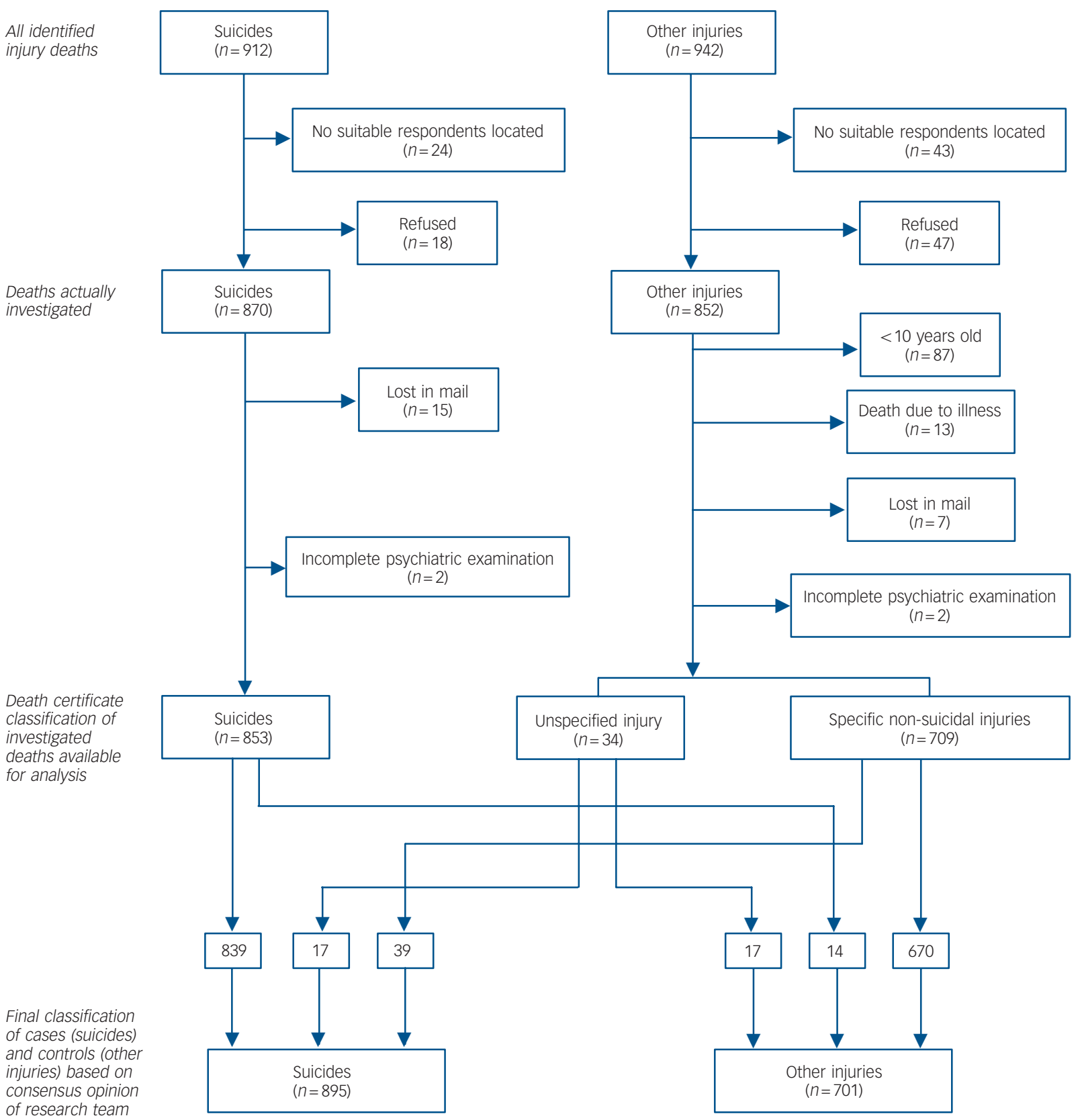

Fig. 1 Identification of individuals who died by suicide and controls.

underestimation of the importance of risk factors for suicide if these factors are more prevalent in the deceased controls than in the general population, but we considered this a less important problem than differential recall biases between proxy informants for suicide decedents and living controls.

Two separate interviews were administered by psychiatrists and other health professionals to a co-resident family member and another close associate of those in both the case group and the control group. The median time between death and interview was 11 months. The survey instrument consists of three components: an audiotaped open-ended interview about the causes and circumstances of death; a structured questionnaire that included information about the circumstances of the death, detailed demographic and socioeconomic status of the decedent, a life-event scale, a quality of life scale, and a medical and psychiatric history; and a semi-structured diagnostic interview.
In the absence of a structured psychiatric examination specific to Chinese respondents, we adapted the Structured Clinical Interview for DSM-IV Axis I disorders (SCID) ${ }^{15,16}$ to generate diagnoses at the time of death, and to make it suitable for use with proxy informants in China, many of whom are illiterate. In non-Western cultures it is particularly important to distinguish depression from normal distress following a serious life event, so interviewers must ensure that the full criteria for depression are present and persist long enough. A related problem is the lack of duration criteria for the diagnosis of adjustment disorder in the DSM system, which could easily result in the application of a psychiatric label to transient dysphoric states. To minimise these problems we added specific culture-sensitive probes for depressive symptoms to increase the instrument's sensitivity to depression and instructed clinical interviewers to use alternative methods of probing for symptoms if respondents had difficulty understanding the standard probes. In accordance with the Chinese diagnostic 
system, ${ }^{17} 1$ month of symptoms was required for a diagnosis of adjustment disorder. We also added some DSM-IV diagnoses not considered in the SCID: mental retardation, dementia, pathological gambling and borderline personality disorder. The interrater reliability of the revised diagnostic instrument was good: the intraclass correlation coefficients for mood disorders, substance use disorders, psychotic disorders, and other mental disorders among 16 coders who independently assessed 37 tape-recorded interviews were $0.87,0.94,0.83$ and 0.71 respectively.

The presence or absence of a mental illness was determined by administering the revised SCID to both the co-resident family member and the close associate of each decedent. If the information provided in these two interviews was different, the case was reviewed in detail by the research team to make a final determination.

\section{Statistical analysis}

The prevalence of most individual mental disorders was too low to permit comparison of rates in the case and control groups across different demographic cohorts, so we combined all specific diagnoses into six diagnostic groups.

(a) Organic mental disorders, including dementia and intellectual disability.

(b) Substance use disorders, including alcohol and other substance use and dependence.

(c) Psychotic disorders - schizophrenia and associated disorders.

(d) Mood disorders, including bipolar disorder, depressive disorder and dysthymic disorder.

(e) Anxiety disorders, including generalised anxiety disorder, panic, phobias, obsessive-compulsive disorder, post-traumatic stress disorder and acute stress disorder.

(f) Other mental disorders, including borderline personality disorder, conversion disorder, hypochondriasis, adjustment disorder, pathological gambling disorder and miscellaneous disorders.

Individuals who had current co-occurring diagnoses from different groups of disorders were coded as having both types of disorders.

Chi-squared tests were used to compare the rates of specific disorders and groups of disorders between the case and control groups. Logistic regression models were used to estimate crude odds ratios, adjusted odds ratios and associated 95\% confidence intervals for suicide for the different diagnostic groups before and after stratification by gender, age group (over or under the median age of 40), and urban (i.e. city and town) versus rural (i.e. village) residence. Only one injury death decedent had an anxiety disorder making it impossible to compute both odds ratios in the stratified cohort analyses, so it was necessary to combine anxiety disorders with other mental disorders in these analyses. The median split was used for age to maximise sample size in the subgroups; a separate analysis was performed comparing rates of any mental disorder in the case group with the controls in six age categories $(10-19,20-29,30-44,45-59$, $60-74,75+)$, but it was not possible to do this for separate diagnostic groups because some age groups had no individuals with the corresponding diagnoses. Comparison of the risk of suicide between cohorts (e.g. comparing the risk of suicide among men with mood disorders to that of women with mood disorders) was assessed by computing the ratio of two adjusted odds ratios (i.e. the odds ratio of suicide among men with mood disorders divided by that for women) and the significance of the ratio was assessed by adding the corresponding interaction term (mood disorder $\times$ gender) to the logistic model. ${ }^{18}$

The study was approved by the institutional review boards of the Beijing Hui Long Guan Hospital and the Chinese Academy of Preventive Medicine.

\section{Results}

Based on DSM-IV criteria, 62.9\% (563/895) of the suicide decedents met criteria for one or more mental disorder at the time of death compared with $17.4 \%(122 / 701)$ of the injury death decedents. Comorbidity of mental disorders (i.e. current occurrence of two or more mental disorders) was more common among suicide decedents than in injury death decedents $(6.3 \% v$. $1.3 \%, \mathrm{OR}=5.3,95 \% \mathrm{CI} 2.6-10.9$ ) and the risk of suicide is greater in those with multiple diagnoses than in those with a single diagnosis (results not shown). As shown in Table 1, with the exception of organic mental disorders, the prevalence of the other five categories of mental disorders is higher in those who died by suicide than in the injury death controls, although the difference is not statistically significant for anxiety disorders because of the small number of individuals. There are significant differences between cases and controls in gender, age group and urban versus rural residence; adjusting for these factors results in an increase in the odds ratio for substance use disorders (primarily alcohol use disorders, which are much more common in male decedents) and a decrease in the odds ratio for psychotic disorders and

Table 1 Comparison of demographic characteristics and prevalence of groups of mental disorders between suicide and non-suicide injuries

\begin{tabular}{|c|c|c|c|c|c|c|c|c|c|c|}
\hline & \multicolumn{2}{|c|}{ Suicide $(n=895)$} & \multicolumn{2}{|c|}{ Injury deaths $(n=701)$} & \multicolumn{2}{|c|}{ Crude odds ratio } & \multicolumn{2}{|c|}{ Adjusted $^{\mathrm{a}}$ odds ratio } & \multicolumn{2}{|c|}{ Multivariate $^{\mathrm{b}}$} \\
\hline & $n$ & $\%$ & $n$ & $\%$ & OR & $95 \% \mathrm{Cl}$ & OR & $95 \% \mathrm{Cl}$ & OR & $95 \% \mathrm{Cl}$ \\
\hline Female & 441 & 49.3 & 169 & 24.1 & 3.06 & $2.46-3.80$ & & - & & \\
\hline Living in rural village & 708 & 79.1 & 506 & 72.2 & 1.46 & $1.16-1.84$ & & - & & \\
\hline Age $>40$ years old & 469 & 52.4 & 327 & 46.6 & 1.26 & $1.03-1.53$ & & & & \\
\hline Any mental disorder & 563 & 62.9 & 122 & 17.4 & 8.05 & $6.35-10.21$ & 9.03 & 7.01-11.64 & & \\
\hline Mood disorders & 356 & 39.8 & 14 & 2.0 & 32.41 & $18.78-55.94$ & 35.19 & $20.24-61.17$ & 44.15 & $25.26-77.18$ \\
\hline Anxiety disorders & 7 & 0.8 & 1 & 0.1 & 5.52 & $0.68-44.95$ & 3.92 & $0.46-33.28$ & 10.39 & $1.20-90.15$ \\
\hline Psychotic disorders & 86 & 9.6 & 14 & 2.0 & 5.22 & $2.94-9.26$ & 4.03 & $2.24-7.26$ & 7.41 & $4.05-13.57$ \\
\hline Substance use disorders & 87 & 9.7 & 56 & 8.0 & 1.24 & $0.87-1.76$ & 1.81 & $1.25-2.61$ & 2.71 & $1.81-4.07$ \\
\hline Organic mental disorders & 47 & 5.3 & 40 & 5.7 & 0.92 & $0.59-1.41$ & 0.95 & $0.61-1.49$ & 1.24 & $0.76-2.04$ \\
\hline Other mental disorders & 39 & 4.4 & 6 & 0.9 & 5.28 & $2.22-12.54$ & 5.53 & $2.28-13.41$ & 10.72 & $4.34-26.49$ \\
\hline
\end{tabular}


anxiety disorders (which are more common in female decedents). All types of disorders except organic mental disorders were significantly related to suicide in the multivariate model: after adjusting for gender, age, urban versus rural residence and other diagnostic groups, the adjusted risk for suicide among people with mood disorders was highest $(\mathrm{OR}=44.2)$ followed by other mental disorders $(\mathrm{OR}=10.7)$, anxiety disorders $(\mathrm{OR}=10.4)$, psychotic disorders $(\mathrm{OR}=7.4)$, substance use disorders $(\mathrm{OR}=2.7)$ and organic mental disorders $(\mathrm{OR}=1.2)$.

Table 2 shows the prevalence of mental disorders by gender in the case group and controls, gender-specific odds ratios for suicide after adjustment for age group and urban versus rural residence, and the male/female ratios of the adjusted odds ratios. There was statistical evidence that the associations of organic mental disorders $(P=0.005)$ and psychotic disorders $(P=0.039)$ with suicide differed in males and females; in both cases associations were approximately three times stronger in males than in females. Despite a 3.6-fold higher risk of suicide in women with mood disorders than in men with mood disorders, this did not achieve statistical significance because of the very wide confidence interval for the odds ratio in female decedents. Substance use disorders (primarily alcohol misuse) were much less prevalent in women than in men, but the risk of suicide associated with substance misuse was the same in women as in men.

Table 3 shows the prevalence of mental disorders by urban versus rural residence, the urban and rural odds ratios after adjusting for gender and age group, and the urban/rural ratios of the adjusted odds ratios. The prevalence of any mental disorder was $25 \%$ higher in urban suicide decedents than in rural suicide decedents and $8 \%$ lower in urban injury decedents than rural injury decedents, so the risk of suicide among individuals with any mental disorder was twice as high in urban versus rural residents $(P=0.008)$. All five categories of mental illness were more prevalent in urban suicide decedents than in rural suicide decedents and - with the exception of substance use disorders much less prevalent in urban versus rural injury decedents, so the odds ratios for suicide in people with these categories of mental disorders were much higher in urban than rural residents. The urban/rural ratio of odds ratios for organic, psychotic, mood and other mental disorders (including anxiety disorders) ranged from 3.8 to 5.8 , but only that for organic mental disorders was statistically significant.

Table 4 shows the prevalence of mental disorders by age group (40 years of age and older $v$. under 40 years of age), the age groupstratified odds ratios after adjusting for gender and urban versus rural residence, and the older/younger ratios of the adjusted odds ratios. The other mental disorders group (including anxiety disorders) was more than twice as prevalent in younger than in older decedents, but the other four categories of disorders were all more prevalent in older than in younger decedents. The risk of suicide among people with substance use disorders was more than twice as high in younger than in older decedents (although the difference was not statistically significant), but the ratios of the odds ratios for the other four categories and for people with any mental disorder was close to 1.0 , indicating that age group (divided at 40 years of age) was not an important moderator of the risk of suicide associated with diagnostic group.

Analysis of the six age groups (10-19, 20-29, 30-44, 45-59, $60-74,75+)$ found that after adjustment for gender and urban versus rural residence, the prevalence of any mental disorder was higher among suicide decedents than among controls in all age strata (data not shown). The adjusted odds ratio for suicide among those with any mental disorder and who were 11-19 years of age was 13.9 (95\% CI 3.1-63.1), for 20 to 29-year-olds it was 18.0 (95\% CI 8.5-30.9), for 30 to 44 -year-olds it was 8.9 (95\% CI 5.6-14.1), for 45 to 59 -year-olds it was 10.4 (95\% CI

Table 2 Comparison of odds ratios (ORs) for suicide between male and female decedents with different mental disorders

\begin{tabular}{|c|c|c|c|c|c|c|c|c|}
\hline \multirow[b]{2}{*}{ Variables } & \multicolumn{3}{|c|}{ Males $(n=986)$} & \multicolumn{3}{|c|}{ Females $(n=610)$} & \multicolumn{2}{|c|}{ Ratio of male/female, OR } \\
\hline & $\begin{array}{c}\text { Suicide, \% } \\
(n=454)\end{array}$ & $\begin{array}{l}\text { Injury, \% } \\
(n=532)\end{array}$ & $\begin{array}{c}\text { Adjusted }^{\mathrm{a}} \text { OR } \\
(95 \% \mathrm{Cl})\end{array}$ & $\begin{array}{c}\text { Suicide, \% } \\
(n=441)\end{array}$ & $\begin{array}{l}\text { Injury, \% } \\
(n=169)\end{array}$ & $\begin{array}{c}\text { Adjusted }^{\mathrm{a}} \text { OR } \\
(95 \% \mathrm{Cl})\end{array}$ & OR & $P^{\mathrm{b}}$ \\
\hline Any mental disorder & 67.4 & 17.5 & $9.64(7.13-13.03)$ & 58.3 & 17.2 & $7.95(5.02-12.59)$ & 1.2 & 0.193 \\
\hline Mood disorders & 43.0 & 2.4 & $28.73(16.05-51.46)$ & 36.5 & 0.6 & $103.78(14.37-749.61)$ & 0.3 & 0.266 \\
\hline Psychotic disorders & 6.4 & 0.8 & $8.97(3.11-25.89)$ & 12.9 & 5.9 & $2.62(1.30-5.31)$ & 3.4 & 0.039 \\
\hline Substance use disorders & 16.3 & 10.0 & $1.79(1.22-2.62)$ & 2.9 & 1.8 & $1.77(0.49-6.31)$ & 1.0 & 0.968 \\
\hline Organic mental disorders & 7.3 & 5.1 & $1.42(0.83-2.41)$ & 3.2 & 7.7 & $0.42(0.19-0.93)$ & 3.4 & 0.005 \\
\hline Other mental disorders ${ }^{c}$ & 4.2 & 0.8 & 7.07 (2.37-21.13) & 6.1 & 1.8 & $3.60(1.07-12.11)$ & 2.0 & 0.514 \\
\hline
\end{tabular}

\begin{tabular}{|c|c|c|c|c|c|c|c|c|}
\hline \multirow[b]{2}{*}{ Variables } & \multicolumn{3}{|c|}{ Urban (town and city) residents ( $n=382)$} & \multicolumn{3}{|c|}{ Rural (village) residents ( $n=1214$ ) } & \multicolumn{2}{|c|}{ Ratio of urban/rural, OR } \\
\hline & $\begin{array}{l}\text { Suicide, } \% \\
\quad(n=187)\end{array}$ & $\begin{array}{l}\text { Injury, \% } \\
(n=195)\end{array}$ & $\begin{array}{l}\text { Adjusted }^{\mathrm{a}} \text { OR } \\
(95 \% \mathrm{Cl})\end{array}$ & $\begin{array}{l}\text { Suicide, } \% \\
(n=708)\end{array}$ & $\begin{array}{l}\text { Injury, \% } \\
(n=506)\end{array}$ & $\begin{array}{l}\text { Adjusted }^{\mathrm{a}} \text { OR } \\
(95 \% \mathrm{Cl})\end{array}$ & OR & $P^{\mathrm{b}}$ \\
\hline Any mental disorder & 74.9 & 16.4 & $15.46(9.15-26.12)$ & 59.8 & 17.8 & $7.58(5.68-10.11)$ & 2.0 & 0.008 \\
\hline Mood disorders & 42.9 & 1.0 & $73.37(17.55-306.74)$ & 39.0 & 2.4 & $28.88(15.84-52.64)$ & 2.5 & 0.190 \\
\hline Psychotic disorders & 12.8 & 1.0 & $10.93(2.49-47.94)$ & 8.8 & 2.4 & $3.06(1.61-5.84)$ & 3.6 & 0.140 \\
\hline Substance use disorders & 11.8 & 12.3 & $1.33(0.70-2.54)$ & 9.2 & 6.3 & $2.13(1.36-3.35)$ & 0.6 & 0.293 \\
\hline Organic mental disorders & 7.0 & 2.6 & $2.50(0.84-7.42)$ & 4.8 & 6.9 & $0.74(0.45-1.23)$ & 3.4 & 0.031 \\
\hline Other mental disorders ${ }^{c}$ & 8.6 & 0.5 & $18.58(2.40-143.94)$ & 4.2 & 1.2 & $3.22(1.30-8.01)$ & 5.8 & 0.136 \\
\hline
\end{tabular}




\begin{tabular}{|c|c|c|c|c|c|c|c|c|}
\hline \multirow[b]{2}{*}{ Variables } & \multicolumn{3}{|c|}{ Decedents $\geqslant 40$ years of age $(n=796)$} & \multicolumn{3}{|c|}{ Decedents $<40$ years of age $(n=800)$} & \multicolumn{2}{|c|}{ Ratio of urban/rural, OR } \\
\hline & $\begin{array}{l}\text { Suicide, } \% \\
\quad(n=469)\end{array}$ & $\begin{array}{l}\text { Injury, \% } \\
(n=327)\end{array}$ & $\begin{array}{l}\text { Adjusted }^{\mathrm{a}} \text { OR } \\
\quad(95 \% \mathrm{Cl})\end{array}$ & $\begin{array}{l}\text { Suicide, } \% \\
(n=426)\end{array}$ & $\begin{array}{l}\text { Injury, \% } \\
(n=374)\end{array}$ & $\begin{array}{c}\text { Adjusted }{ }^{\mathrm{a}} \text { OR } \\
(95 \% \mathrm{Cl})\end{array}$ & OR & $P^{\mathrm{b}}$ \\
\hline Any mental disorder & 69.3 & 20.2 & $9.14(6.51-12.84)$ & 55.9 & 15.0 & $9.13(6.24-13.35)$ & 1.0 & 0.335 \\
\hline Mood disorders & 45.8 & 2.8 & $30.92(15.51-61.67)$ & 33.1 & 1.3 & $41.34(16.47-103.73)$ & 0.7 & 0.857 \\
\hline Psychotic disorders & 11.1 & 2.5 & $4.20(1.95-9.05)$ & 8.0 & 1.6 & $4.35(1.73-10.95)$ & 1.0 & 0.776 \\
\hline Substance use disorders & 10.0 & 9.5 & $1.30(0.80-2.12)$ & 9.4 & 6.7 & $2.78(1.59-4.84)$ & 0.5 & 0.272 \\
\hline Organic mental disorders & 6.6 & 6.4 & $1.04(0.58-1.86)$ & 3.8 & 5.1 & $0.86(0.42-1.76)$ & 1.2 & 0.609 \\
\hline Other mental disorders ${ }^{c}$ & 3.4 & 0.6 & $5.29(1.19-23.52)$ & 7.0 & 1.3 & $5.45(2.01-14.76)$ & 1.0 & 0.969 \\
\hline
\end{tabular}

5.9-18.3), for 60 to 74-year-olds it was 13.7 (95\% CI 6.8-27.8), and for people 75 or older it was 3.0 (95\% CI 1.4-6.4). These results suggest that mental illnesses are more important risk factors for suicide in young adults than in older people, but despite the sixfold range in the adjusted odds ratios $\times$ age group (3.0 to 18.0) the multivariate analysis did not find statistically significant differences in the risk $\times$ age group, primarily because of the wide confidence intervals associated with the estimated odds ratios.

\section{Discussion}

\section{Main findings}

In accordance with our a priori hypothesis, this study found that both the prevalence of different categories of mental disorders among suicide decedents and the risk of suicide among people with different types of mental disorders vary across demographic groups and by urban versus rural residence. Previous studies have suggested that risk factors for suicide may not be uniform in all population cohorts. ${ }^{19-21}$ We have advanced this discussion by comparing cohort-specific odds ratios in different categories of mental disorders for three factors that are potential moderators of the risk of suicide - gender, age and urban versus rural residence.

The overall prevalence of mental disorders in the suicide decedents $(63 \%)$ is substantially lower than the prevalence reported in Western psychological autopsy studies ${ }^{2,3,6}$ but somewhat higher than that reported by another psychological autopsy study in China, ${ }^{22}$ which used the SCID to assess young rural suicide decedents and found that only 55\% of male decedents and $39 \%$ of female decedents had a mental illness at the time of the suicide. The reasons for the lower prevalence of mental disorders in Chinese suicide decedents are unknown but we believe it is related to higher levels of impulsive behaviour combined with easy access to highly lethal means (i.e. pesticides), particularly in rural areas.

Despite the lower prevalence, the relative importance of different classes of mental disorders as risk factors for suicide found in this study is comparable to that seen in high-income countries. Similar to other studies, ${ }^{6,8}$ mood disorders are, by far, the most important category of mental disorders. The relatively less important (although statistically significant) role of substance use disorders seen in this study compared with that reported in high-income countries ${ }^{6,23}$ is partly due to the lower prevalence of these conditions in China (although rates of alcohol misuse have been increasing recently). ${ }^{14,24,25}$ Another possibility is that higher rates of substance misuse in accidental death decedents than in the general population could result in an underestimate of the odds ratio for substance misuse in suicide. Given the similar prevalence of substance use disorders reported in males in the general population ${ }^{14}$ to that found in our male accidental decedents (11.3\% v. 10.0\%), this does not appear to be the case for men. However, the prevalence of substance misuse in female accidental decedents $(1.8 \%)$ is substantially higher than reported in females in the general population ${ }^{14}(0.3 \%)$, so the reported odds ratio for substance misuse in females (1.77) may be an underestimate.

Our results highlight the important distinction between population-based attributable risk and individual-based clinical risk. For example, we found that the prevalence of psychotic disorders in female suicide decedents is double that in male suicide decedents $(12.9 \%$ v. $6.4 \%)$, so psychotic disorders are a relatively more important cause of suicide in women than in men. ${ }^{26}$ But, similar to other studies in China, ${ }^{27}$ we found that the risk of suicide among males with psychotic disorders is 3.4-fold higher than that of females with psychotic disorders so clinicians need to have a greater 'index of suspicion' for males with psychotic disorders. Thus the target of universal preventive steps aimed at the general population may need to be different from the focus for selective and indicated interventions and treatments aimed at high-risk groups and individuals.

The most important specific finding was that mental illnesses are much less important as risk factors for suicide among rural residents than among urban residents. The adjusted odds ratio for suicide for rural residents with any mental disorder was 7.6 compared to an odds ratio of 15.5 for urban residents with mental disorders $(P=0.008)$. Previous reports from China have shown major differences in the characteristics of urban versus rural suicide decedents ${ }^{12}$ and the important role of impulsivity in the suicides of both men and women from rural communities. ${ }^{28}$ The current analysis found that, with the exception of substance use disorders (which conferred a greater risk of suicide among rural residents), the risk of suicide among individuals with mood disorders, psychotic disorders, organic mental disorders or other mental disorders (including anxiety disorders) living in urban areas was more than double the risk among individuals with these disorders living in rural areas. The prevalence of mental disorders in rural versus urban injury death decedents was similar $(17.8 \% v$. $16.4 \%$ ), so it is unlikely that the much lower risk of suicide among rural residents with mental disorders is the result of underdiagnosis in rural decedents. Nor is it likely that the lower suicide risk associated with mental illness is the result of better services and support for the mentally ill in rural areas, many of which lack any mental health services whatsoever. We believe this is a robust finding with important theoretical and practical implications.

Urban versus rural residence is a composite indicator that captures a range of social and contextual variables relevant to suicide (e.g. economic and educational status, density of family relationships, employment, access to means of suicide, access to 
health resources), but it is not included in most multidimensional models of suicide, ${ }^{9,10}$ possibly because these models are primarily based on work in high-income countries where a much smaller proportion of the population live in rural areas and, perhaps, where the urban-rural divide is not as pronounced as in low- and middle-income countries. Based on our findings, we hypothesise that urban versus rural residence explains a substantial proportion of the within-country variance in suicide rates in low- and middle-income countries with large agriculture-based populations and that it is a more powerful predictor of suicide rates than its component variables (e.g. economic level, education, employment). The relationship of urban versus rural residence to suicide risk may become attenuated with urbanisation and with high rates of rural-to-urban work migration, so eventually it may be more fruitful to dissect it into its component variables. But even as the urbanisation process progresses, major urban versus rural differences in social structure and healthcare systems will persist, so it will remain an important dimension that is relevant to the provision of preventive and treatment services for suicide.

\section{Implications}

Our results suggest that the network of causal factors leading to suicide in rural areas is substantially different than that in urban areas. The ready availability of potent pesticides in many rural households may increase the likelihood that impulsive, low-intent suicidal acts following intense interpersonal conflicts among people without a diagnosable mental illness result in death. ${ }^{22}$ If this is the case, then the focus of preventive efforts in rural areas should be on providing educational and social interventions aimed at improving coping skills and conflict resolution skills in community members; and on decreasing access to pesticides, reducing the lethality of pesticides, promoting public health campaigns about the safe use and storage of pesticides, and improving the treatment of pesticide poisoning. ${ }^{29}$ The lack of adequate mental health services in rural areas is a common problem in low- and middle-income countries that urgently needs to be addressed, ${ }^{30}$ but improving rural mental health services should only be one component - and not necessarily the most important component - of suicide prevention efforts in these countries.

\section{Strengths and limitations}

Several factors need to be taken into consideration when interpreting these results. The demographic profiles of mental disorders ${ }^{14}$ and of suicides in China ${ }^{4,5}$ are quite different from that reported in most high-income countries, so the relationship between suicide risk, diagnostic groups and the three factors considered (gender, age and urban versus rural residence) may be different in China than elsewhere.

Despite using data from the largest case-control psychological autopsy yet reported, the low prevalence of some mental disorders in the injury death control group made it impossible to conduct some of the stratified analyses and resulted in large confidence intervals around some of the estimated odds ratios, increasing the risk of type II errors (i.e. some important differences did not meet the criteria of statistical significance). Overcoming this problem will require conducting meta-analyses that combine results from several large psychological autopsy studies that have used comparable methods.

As a result of the sample size considerations we arbitrarily dichotomised age by the median age and found few differences in the risk of suicide associated with different mental disorders between persons under or over 40 years of age. Our analysis by six age groups found age-related differences in risk of suicide among people with any mental disorder when comparing more narrowly defined age ranges, but the numbers of individuals in the age-specific cohorts were too few to reach statistical significance. There may also be important diagnostic groupspecific differences in suicide risk in different age groups that we were unable to assess.

There are several other demographic and clinical factors which may moderate the relationship of suicide risk and mental illnesses that we did not consider: for example, education, income, marital status, prior suicide attempt, and family history of suicide. Given the practical problems associated with travelling to 23 sites around the country, the time from death to interview (median 11 months) was longer than in most psychological autopsy studies; ${ }^{3,6}$ but there was no significant difference in the time to interview between those in the case group and the controls, and the prevalence of mental disorders was similar in cases interviewed early and those interviewed later, so it is unlikely that the relatively long time to interview affected the reported results.

As discussed in the Method, the use of deceased controls has the disadvantage that potential risk factors for suicide may also be risk factors for other causes of death that could lead to an underestimation of the importance of such factors. But the overall prevalence of mental disorders among men and women in the injury death group (17.5\% and $17.2 \%$ respectively) are similar to those found in a large community-based epidemiological study in four Chinese provinces that used similar diagnostic methods ${ }^{14}$ (20.1\% and $14.8 \%$ respectively), so it does not appear that accidental death decedents have unusually high rates of mental illness that would obscure the importance of mental illnesses in suicide.

The specific findings of this analysis may or may not hold true in other countries where the characteristics of suicide and the demographic profile of mental illnesses are different. Similar analyses of large case-control psychological autopsy studies are needed to assess the generalisability of our specific findings. The core finding that we believe to be widely relevant is that the importance of mental illnesses as risk factors for suicide varies widely both by the type of mental illness and by demographic cohort. We also believe it essential to consider urban versus rural residence as a key variable in the understanding and management of suicide, particularly in low- and middle-income countries.

\footnotetext{
Yongsheng Tong, MD, WHO collaborating Center for Research and Training in Suicide Prevention, Beijing Suicide Research and Prevention Center, Beijing Hui Long Guan Hospital, China; Michael R. Phillips, MD, MA, MPH, WHO Collaborating Center for Research and Training in Suicide Prevention, Beijing Suicide Research and Prevention Center, Beijing Hui Long Guan Hospital, China, and Departments of Psychiatry and Global Health, Emory University, Atlanta, Georgia, USA

Correspondence: Michael R. Phillips, Executive Director WHO Collaborating Center for Research and Training in Suicide Prevention, Beijing Suicide Research and Prevention Center, Beijing Hui Long Guan Hospital Beijing 100096, China. Email: phillipschina@yahoo.com

First received 6 Jul 2009, final revision 30 Jan 2010, accepted 9 Feb 2010
}

\section{Funding}

This study is part of the 'Causes and Prevention of Accidental Deaths in China' project, which was supported by grants from the Ford Foundation, the Save the Children Fund, and Befrienders International.

\section{Acknowledgements}

Participating institutions include 23 disease surveillance points and four psychiatric centres (Department of Neuropsychiatry, Xijing Hospital, Xian, Shaanxi Province; Jingzhou City Psychiatric Hospital, Hubei Province; Shenyang Mental Health Centre, Liaoning Province; and Suzhou Guangji Hospital, Jiangsu Province). The authors thank all the institutions, and Suzhou Guangji Hospital, Jiangsu Province). The authors thank all the institutions,
investigators and respondents for their contribution to the research, and David Gunnell and Morton Silverman for comments on earlier versions of the manuscript. 


\section{References}

1 Appleby L, Dennehy JA, Thomas CS, Faragher EB, Lewis G. Aftercare and clinical characteristics of people with mental illness who commit suicide: a case-control study. Lancet 1999; 353: 1397-400.

2 Arsenault-Lapierre G, Kim C, Turecki G. Psychiatric diagnoses in 3275 suicides: a meta-analysis. BMC Psychiatry 2004; 4: 37.

3 Mortensen PB, Agerbo E, Erikson T, Qin P, Westergaard-Nielsen N Psychiatric illness and risk factors for suicide in Denmark. Lancet 2000; 355 9-12.

4 Phillips MR, Li X, Zhang Y. Suicide rates in China, 1995-99. Lancet 2002; 359 835-40.

5 Phillips MR, Yang G, Zhang Y, Wang L, Ji H, Zhou M. Risk factors for suicide in China: a national case-control psychological autopsy study. Lancet 2002; 360: $1728-36$

6 Wærn M, Runeson BS, Allebeck P, Beskow J, Rubenowitz E, Skoog I, et al Mental disorder in elderly suicides: a case-control study. Am J Psychiatry 2002; 159: 450-5.

7 Klick J, Markowitz S. Are mental health insurance mandates effective? Evidence from suicides. Health Econ 2006; 15: 83-97.

8 Ran MS, Wu QH, Conwell Y, Chen EY, Chan CL. Suicidal behavior among inpatients with schizophrenia and mood disorders in Chengdu, China. Suicide Life Threat Behav 2004; 34: 311-9.

9 Maris RW. Suicide. Lancet 2002; 360: 319-26.

10 Wasserman D. A stress-vulnerability model and the development of the suicidal process. In Suicide: An Unnecessary Death (ed D Wasserman): 13-27. Martin Dunitz, 2001

11 Phillips MR, Liu HQ, Zhang YP. Suicide and social change in China. Cult Med Psychiatry 1999; 23: 25-50.

12 Yang G, Phillips MR, Zhou M, Wang L, Zhang Y, Xu D. Understanding the unique characteristics of suicide in China: national psychological autopsy study. Biomed Environ Sci 2005; 18: 379-89.

13 Hawton K, Appleby L, Platt S, Foster T, Cooper J, Malmberg A, et al The psychological autopsy approach to studying suicide: a review of methodological issues. J Affect Disord 1998; 50: 269-76.

14 Phillips MR, Zhang JX, Shi QC, Song ZQ, Ding ZJ, Pang ST, et al. Prevalence, associated disability and treatment of mental disorders in four provinces in China, 2001-2005: an epidemiological survey. Lancet 2009; 373: 2041-53.

15 First MB, Spitzer RL, Gibbon M, Williams JB. Structured Clinical Interview for DSM-IV Axis I Disorders. Biometrics Research Department, New York State Psychiatric Institute, 1996.
16 American Psychiatric Association. Diagnostic and Statistical Manual of Mental Disorders (4th edn) (DSM-IV). APA, 1994.

17 Chinese Society of Psychiatry, Chinese Medical Association. Chinese Classification of Mental Disorders and Diagnostic Criteria (revised 2nd edn) (CCMD-2-R) [in Chinese]. Southeast University Publishing House, 1995.

18 Altman DG, Bland JM. Interaction revisited: the difference between two estimate. BMJ 2003; 326: 219.

19 Hunt I, Kapur N, Webb R, Robinson J, Burns J, Turnbull P, et al. Suicide in current psychiatric in-patients: a case-control study, the national confidential inquiry into suicide and homicide. Psychol Med 2007; 37: 831-7.

20 Modai I, Kuperman J, Goldberg I, Goldish M, Mendel S. Suicide risk factors and suicide vulnerability in various major psychiatric disorders. Med Inform 2004; 29: 65-74.

21 Zivin K, Kim M, McCarthy JF, Austin KL, Hoggatt KJ, Walters H, et al. Suicide mortality among individuals receiving treatment for depression in the veterans affairs health system: associations with patient and treatment setting characteristics. Am J Public Health 2007; 97: 2193-8.

22 Zhang J, Wieczorek W, Conwell Y, Tu XM, Wu BYW, Xiao S, et al. Characteristics of young rural Chinese suicides: a psychological autopsy study. Psychol Med 2010; 40: 581-9.

23 Cherpitel CJ, Borges GL, Wilcox H. Acute alcohol use and suicidal behavior: a review of the literature. Alcohol Clin Exp Res 2004; 28: 18-28s.

24 Hao W, Young DS, Xiao SY, Li L, Zhang YL. Alcohol consumption and alcoholrelated problems: Chinese experience from six area samples, 1994. Addiction 1999; 94: 1467-76.

25 Hao W, Su ZH, Liu BL, Zhang K, Yang HQ, Chen SZ, et al. Drinking and drinking patterns and health status in the general population of five areas of China. Alcohol Alcohol 2003; 39: 43-52.

26 Phillips MR, Yang GH, Li SR, Li Y. Suicide and the unique prevalence pattern of schizophrenia in mainland China: a retrospective observational study. Lancet 2004; 364: 1062-8

27 Ran MS, Chen EYH, Conwell Y, Chan CLW, Yip PSF, Xiang MZ, et al. Mortality in people with schizophrenia in rural China: 10-year cohort study. $\mathrm{Br}$ Psychiatry 2007; 190: 237-42.

28 Conner KR, Phillips MR, Meldrum S, Knox KL, Zhang Y, Yang G. Low-planned suicides in China. Psychol Med 2005; 35: 1197-204.

29 Gunnell D, Eddleston M, Phillips MR, Konradsen F. Worldwide patterns of fatal pesticide self-poisoning. BMC Public Health 2007; 7: 357

30 Lancet Global Mental Health Group. Scale up services for mental disorders: a call for action. Lancet 2007: 370: 1241-52.

\section{0 words}

\section{A psychiatrist is . . .}

\section{Nick Craddock}

a medical specialist who helps people with abnormal thinking, mood and behaviour. Aware of the importance of developmental context and psychological, social and cultural factors, the psychiatrist brings particular knowledge of biological factors, including somatic illness, to the multi-professional team. Embracing complexity, the psychiatrist is a critical but pragmatic thinker who seeks evidence, advocates research and uses comprehensive diagnosis to guide effective risk assessment and treatment. Acknowledging the important contributions of non-medical colleagues, and aware of the limitations of medical approaches, the psychiatrist provides clinical leadership, teaching and supervision when required, always placing patient interests at the heart of actions. 